\title{
SHADOW-CASTING: A TECHNIQUE TO SHOW SURFACE TEXTURE IN MICRO- SCOPICAL MATERIAL
}

WILFRID TAYLOR DEMPSTER AND ROBLEY COOK WILLIAMS

Department of Anatomy, and Department of Physics, University of Michigan, Ann Arbor

TWO TEXT Figures and ONe Plate (NINe Figures)

Objects seen through the microscope by transmitted light are translucent in differing degrees. Staining gives a measure of differential opacity and contrast to biological material but, even here, the appearance, like that of an $\mathrm{x}$-ray picture, is a record of relative opacities. Varying the focal plane, of course, allows a range of optical sections, and from this the surface appearance can be inferred to some extent. Surfaces are actually seen with the microscope when opaque objects are viewed by oblique illumination, but this method is illadapted for the higher powers of microscopical work and is little used in standard microscopy save as an occasional adjunct or for special purposes where low powers suffice.

The method presented here allows surface texture to be detected over the whole range of magnification of the microscope. The technique was originally developed for work with the electron microscope (Williams and Wyckoff, '44) as a means of measuring thickness of objects and of increasing image contrast. Williams and Wyckoff also applied it in revealing the surface detail of bacteria ('45a), viruses ('45b, c, d) and minute chemical aggregations ('45e). A special "replica" method using colloidin films was developed for use on opaque material so that metal and mineral surfaces could be studied ('46). Through use of the technique in electron microscopy, features far beyond the range of resolution of the light microscope have been revealed. 
INTERPRETATION OF SHADOWED MATERIAL

The method consists simply of depositing in vacuo a film of metallic chromium (or other suitable metal) on a specimen and its substrate at an oblique angle. Chromium is vaporized from a heated filament in a suitable vacuum, and particles of atomic chromium radiate away in straight line paths. The chromium falling on the specimen sticks and builds up most thickly on surfaces exposed most directly to the filament, that is, on surfaces perpendicular to lines of radiation. On oblique surfaces the deposit is thinner, and it is absent from regions obscured or "shadowed" by the specimen. The distribution of chromium is like that of light from a single point source falling obliquely on an object; highlights appear on the near face and a shadow is present on the far side away from the light. Intermediate areas of the surface show gradations of light which are due to variations in surface texture.

On shadow-cast objects viewed with the light microscope in transmitted light, the heavier chromium films block the light, while the "shadows" appear transparent. Thus, the appearance is the reverse of ordinary light and shadow pictures; highlights are dark and shadows are light (fig. 1B and D). After a little experience with the microscope, one compensates automatically for the reversed values much as he does in writing on a blackboard. In photomicrographic negatives the highlights and shadows have a natural appearance (fig. 1A and $\mathrm{C}$ ). The latter figures and those of the accompanying piate also are paper prints made from lantern slide positives which in turn were made from photomicrographic negatives.

The observation of shadow-cast material with vertical illuminators was tried to see if the natural shadow appearance could be obtained directly, but internal reflections from the slide and obliquely incident rays through the objective resulted in a glaring background that destroyed the shadow picture.

The metallic film deposited in the technique is without visible texture and it is only when the deposit falls on an ir- 
regular or undulating surface that variations are detectable. Since the ordinary thickness of deposit found to give good results is only $200 \AA$ (0.02 micron), any distortion due to deposit thickness is far below the range of visible detection. Even with the electron microscope, this thickness of chromium allows magnifications up to 10,000 without evident distortion.

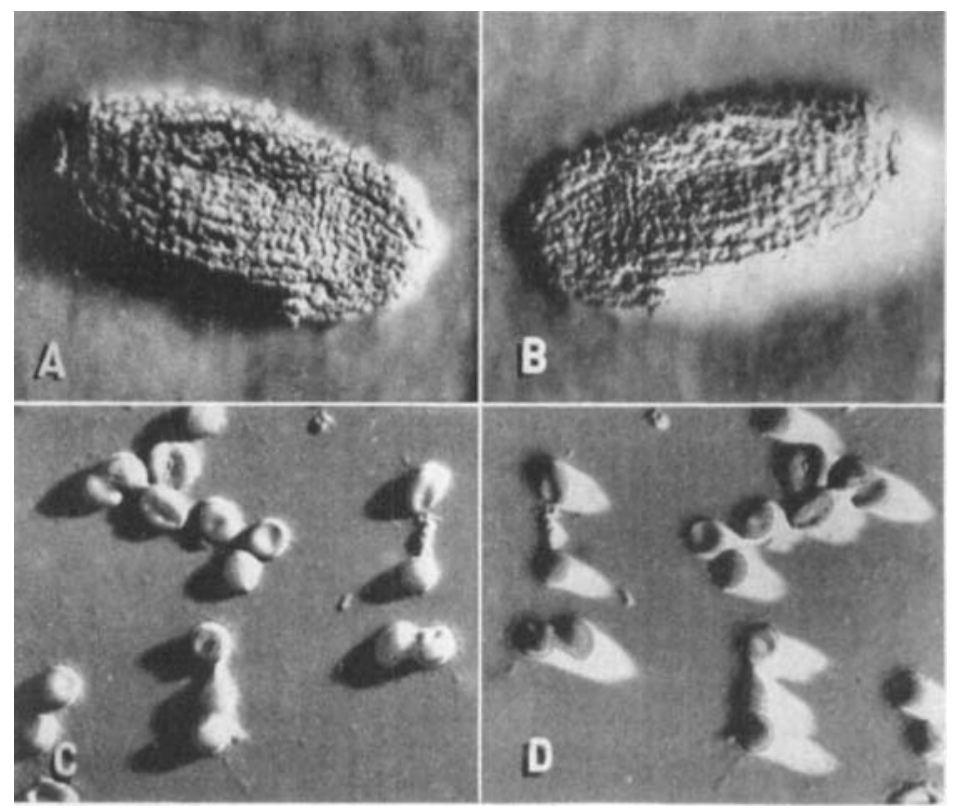

Fig. 1 Photographs at the left show natural shadows as seen in photo negatives. The specimens appear to be lighted from the right and above, and shadows fall toward the left. $A$. is a Paramoecium dried to a cover slip. $\times 750$. C. shows human erythrocytes. $\times 750$. Photographs B. and D., at the right, are mirror images having the appearance of the actual specimens or of positive prints.

\section{EXAMPLES AND PREPARATION}

Representative samples of shadow-cast material viewed with the light microscope are assembled (figs. 3-11) in the accompanying plate. These will serve as a basis for an account of our experience with shadowed material and the visual microscope. Blood smears (figs. 3, 8 and 9) were made in the usual way and with no. 1 cover slips; a drop of blood was 
placed between two covers which were then sheared apart and dried. For figure 1C, a drop of blood and a drop of osmic acid were mixed between cover glasses for several minutes before shearing apart and washing in water. After drying thoroughly, the cover slips with the dried blood films were stored in a desiccator jar until they could be shadowed conveniently (by the procedure to be outlined).

In figure 3 , the erythrocytes shown are flattened discs. Most show a central depression suggestive of the biconcave shape of the cells. In two or three cells which overlap others, the overlap is apparent by a raised area of the exposed surface. Blood cell plasticity is suggested by the irregular shapes of the cells where they were wedged together during drying. The cells are with few exceptions smooth surfaced and the circumference is sharp edged, presumably due to drying. In a few cells, the edge is irregular.

White cells (not shown) flatten to the same range of thickness as the red cells. In granulocytes, the cell membrane flattens on the cell contents and the surface is thrown into a bumpy texture corresponding with separate and grouped granules of a more resistant nature than the general cytoplasm. Lobation of polymorphonuclear nuclei is evident through bulging of the flattened surface.

In figure 8 , the blood film was thick, and drying was slow. Many of the erythrocytes were in rouleaux. The cells generally have a velvety texture. In some cells, surfaces and edges have a disintegrative appearance. Edges are typically rounded and the cells lack the crisp appearance associated with quick drying and thinner films. Though the concave central region is often seen, the cells have a puffy appearance when compared with the more quickly dried cells.

After fixation with osmic acid (fig. 1c), cell areas are smaller and the cells are thicker than in air dried films. Though cells occasionally show a slight surface roughening, most have smooth surfaces. A central depression is generally evident. A somewhat swollen character seems to result from this fixation. Some cells show circumferential ridges due to 
drying. Irregular granules and blotches in the background are probably blood platelets.

Figure 9 shows chicken blood from birds infected with malaria (Plasmodium gallinaceum). Only the upper left corpuscle appears normal. In the flattened cell, the nucleus stands out as a round elevation. Cell circumferences show a drying ridge. The various cells show mottled irregular surfaces and from one to about four depressed pits of varying shapes. The collapse of vacuoles in the infective organisms within the blood cells no doubt accounts for the moth-eaten irregularities of the surface.

Figures 4, 5 and 6 showing spermatozoa are from Ringersfluid suspensions of sperm from the epididymus or testes. Smears were simply air dried prior to shadow-casting. Sperm smears were also fixed by several standard fixing agents and were washed prior to drying. The English sparrow sperm of figure 6 was treated with saturated sublimate. Fixation ordinarily precipitates proteins from the sperm medium and photographs of shadowed material have a dirty appearing background. If two cover slips are pinched tightly together by forceps, the edges may be touched to a sperm suspension; capillary action draws the fluid up between the cover slips. When the slips are sheared apart sideways, the uppermost ends of the cover slips have a cleaner background than the lower. After drying (or fixation), immersion of the cover slips in water for a half minute or so will redissolve and clear away any salts that may have crystallized from the Ringer's fluid. The material is then dried again before shadow-casting.

Dog sperm (fig. 4) shows the typical flattened head, a slight constriction at the neck and a gradually tapering tail. Mouse sperm (fig. 5), a much larger sperm, shows the peculiar hooked and eccentric head and the gradually tapering tail. Approximately half of the tail is figured. The English sparrow sperm (Passer domesticus) is a fine tapering rod. The anterior end (fig. 6) shows a spiralling envelope (three spirals to the thickest part of the head) which rapidly decreases to a thin spiralling membrane that continues over the whole length of 
the filament. Human sperm (not shown) shows an oval shaped head, flattened anteriorly and bulbous behind. A midpiece is not well-differentiated from the generally tapering tail.

Five to ten micron sections of various tissues prepared by the standard paraffin technique could be studied after shadowcasting. The sections from the microtome were merely flattened and affixed to no. 1 cover slips, and after drying, the paraffin was dissolved away through immersion in carbon bisulphide, benzene and ether. Then, after evaporation of the solvents, the material was shadow-cast.

Parts of several muscle fibers in longitudinal section are shown in figure 7. Myofibrils show in relief and sarcomeres separated by $Z$ stripes (and other aspects of the striation pattern as well) are easily recognized.

Figure 10 shows part of a section of pulmonary lymph node. Most of the cells, such as two to the right of "10," are macrophages; they appear superficially like "popeorn kernels" with numerous irregular processes. In the fixed preparation, they are not flattened to the cover slip like cells in smears. The macrophages contained many black carbon particles and these show dark in positive prints of shadow-cast material. In the negative appearance shown in the plate, however, the carbon shows white. One may, however, distinguish ordinary highlights due to modelling from the white areas representing carbon. Such reversal of tone as found with carbon granules is no problem.

Attempts were made to photograph shadow-cast material that had been stained in the routine way for transmitted light microscopy. In the negative, where shadow-cast material by itself looks three-dimensional, the values of stained preparations are reversed. The resultant photographs of stained and shadow-cast material unlike the carbon pictures above are confusing. The method, nevertheless, might be useful for visual work where color and tone may be differentiated by the eye. If such material is lightly stained, it may then be photographed with appropriate color filters and the typical shadow- 
cast appearance is shown without the confusion of superimposed tones due to staining.

It should be mentioned that because of the contrast of shadowed material, photomicrography is on the whole easy. Filters or color sensitive plates are not required as a routine. Oil-immersion photomicrography, however, is complicated by the low depth of focus of the objectives used, and suitable material must be thin.

All of the preceding figures are from oil-immersion photographs. In contrast, figure 11 is a low-power photograph which shows a general view of decalcified bone and cartilage cartilage (to the left) and bony trabeculae (to the right) are seen. The coarse granular areas between bony spicules are bone marrow. The general cartilage matrix shows finely granular. Both individual cartilage lacunae with included cells and rows of lacunae are shown. Eroding tongues of marrow work into the cartilage rows at one or two places. A lamellar structure shows in the fibrous framework of the bone trabeculae and occasional osteocytes are seen.

\section{TYPICAL APPLICATIONS OF THE TECHNIQUE}

It has been our purpose to show that usable and often striking pictures of microscopic surfaces and textures may be secm with the shadow-easting technique. This is a technique which not only shows the whole range of appearance from low power to oil-immersion, but which is extended through the range of magnification of the electron microscope as well. For the electron microscope, however, a special substrate of thin colloidin must be used in place of glass cover slips. Though the depth of focus with the latter instrument is greater than with oil-immersion, the opacity of thick material to electrons limits the instrument to very thin material. Thus, there probably are only a few types of material that may be studied first by one type of microscopy and then by the other. Material suitable for electron microscopy and mounted on colloidin for shadow-casting may, of course, be viewed with the light microscope. Some adaptation of the replica method, ('46), to 
biological material might extend the range. In both electron and light microscopy, the shadow technique produces the same shadow appearance and shows surfaces and texture in the same way.

Microscopic heights may be measured from shadow lengths when the casting angle is determined accurately. Visual and photographic contrast is a positive advantage of the technique. The contrast varies with the amount of metal deposited. If a shadow-cast specimen, on preliminary examination, is not contrasty enough, it may be reshadowed by a further deposit if the specimen is oriented so that the second shadow corresponds with the first. As mentioned above, the light staining of specimens may be an advantage in visual work for simultaneous study of optical sections and surface textures. Part of a specimen may be covered by paper or by a fenestrated grill during the shadowing process and the resultant preparation will serve for side-by-side study of surfaces and interior views. The foregoing figures on human blood show striking differences in appearance due to drying. effects and fixation - changes more apparent than ordinarily seen in microscopy. It would appear that various fixations could be compared and contrasted for the evaluation of standard techniques and that the effects of many experimental procedures could be studied in terms of surface textures. Though the present technique is limited to desiccated material, routine permanent preparations in balsam are too.

When the shadow-casting apparatus is once set up for use, the casting technique is probably simpler and quicker than many of the routine techniques of staining. Number 1 cover slips are advisable whenever oil-immersion work might be desired. We have affixed material to cover slips rather than to slides and after shadow-casting, the slips were mounted face down on a slide with balsam or clarite. No further procedure was required. Before casting, material was accumulated in a desiccator jar with suitable precautions for avoiding dust and dirt. 


\section{APPARATUS AND METHOD}

In order to carry out the procedures of shadow-casting, it is necessary to have a vacuum equipment similar to that shown in figure 2. The essential parts are a glass bell-jar and a base-plate provided with vacuum tight electrical leads. The degree of vacuum required (about $10^{-4} \mathrm{~mm}$ of $\mathrm{Hg}$ ) is such that a diffusion pump as well as a mechanical pump must be used.

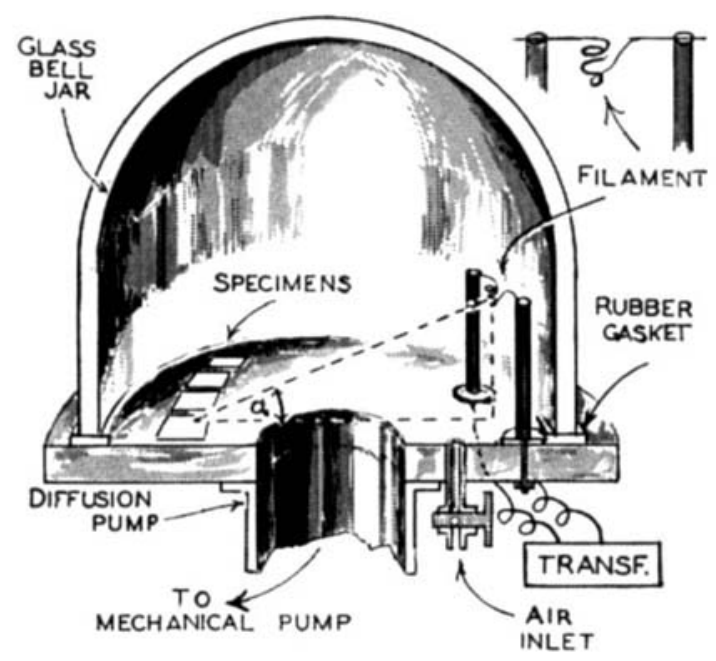

Fig. 2 Diagram of the vacuum chamber required for shadow-casting. The distance from the filament to the spceimens, the shadow-easting angle " $a$," and the amount of metal vaporized from the filament are determined for each run; otherwise, the procedure is routine.

The filament of 20 mil. tungsten wire is coiled as shown in the figure - the wire is wound around a wood screw to form a basket - and is mounted between two electrode posts. Chromium is ordinarily used as the metal to be deposited and the computation of the amount to be used is indicated below. Weighed pieces of chromium metal are placed in the filament; the cover slips are arranged face up and in a semicircle as shown. After the bell-jar is placed in position, the pumps are started. With reasonably fast pumps (Megavac plus a $t^{\prime \prime}$ oil-diffusion pump), a good enough vacuum is obtained in 
about 15 minutes. The filament is then operated at about 10 volts and 20 amperes until the chromium is all evaporated. Air is then admitted to the chamber and the shadowed specimens are ready for mounting and examination. Care must be taken to make sure that all material is dry before insertion into the chamber.

The mass of chromium to be evaporated is given by the formula :

$$
M=\frac{4 \pi r^{2} t D}{\tan a} \text { where }
$$

$\mathrm{M}$ is the mass in grams, $\mathrm{r}$ is the distance from the filament to the specimens, $t$ is the thickness of metal film to be deposited, $D$ is the density of the metal, and $a$ is the angle shown in figure 2 .

A convenient value for $\mathrm{r}$ is $15 \mathrm{~cm}$, and a satisfactory film thickness is 0.02 micron. For the case of chromium (density 7 ) and an angle of $15^{\circ}$ for a, the computed mass to use is $70 \mathrm{mg}$.

\section{SUMMARY}

Shadow-casting is a technique which shows the surface texture of microscopical material rather than the routine transparent appearances. Sections or smears may be studied throughout the whole range of microscopical magnification. The method involves the in vacuo deposition of a metallic film on dried specimens. Metal is deposited from an oblique angle so that it coats some surfaces of specimens more than others. Highlights and shadows are brought out and the material shows in relief. Illustrative photomicrographs, the range of application of the method and details of the apparatus and procedure are considered.

\section{LITERATURE CITED}

Wilitams, R. C., AND R. W. G. Wyckoff $19+4$ The thickness of electron microscopic objects. J. A ppl. Physics, vol. 15, pp. 712-716.

1945a Shadowed electron micrographs of bacteria. Proc. Soc. Exp. Biol. and Med., vol. 59, pp. 265-270.

1945b Electron shadow-micrography of virus particles. Proc. Soc. Exp. Biol. and Med., rol. 58, р. 265-270. 
Williams, R. C., AND R. W. G. WyCKoFr 1945e Electron shadow micrography of the tobaceo mosaic virus protein. Science, vol. 101, pp. 594-596. 1945d The electron micrography of crystal line plant viruses. Science, vol. 102, pp. 277-278.

1945e Electron shadow-micrographs of haemocyanin molecules. Nature, vol. 156, p. 68.

1946 Applications of metallic shadow-casting to microscopy. J. Appl. Physics, vol, 17, pp. 23-33. 


\section{PLATE 1}

EXPLANATION OF FIGURES

3 Unfixed and dried human erythroeytes. $\times 1000$.

4 Unfixed and dried dog sperm. $\times 1000$.

5 Anterior part of mouse sperm. $\times 1000$.

6 Dried English sparrow sperm. $\times 1000$.

7 Parts of three voluntary musele fibers showing striations, from a longitudinal paraffin section through monkey diaphragm. $\times 1000$.

8 Human erythrocytes from a thick region of a smear, unfixed. $\times 1000$.

9 Chick erythrocytes showing changes due to malaria infection. A normal cell is at the upper left. $\times 1000$.

10 A group of macrophages from a paraffin section of a pulmonary lymph node. Included carbon granules show white. $\times 1000$.

11 A low power photomicrograph of a section of monkey vertebrae, $\times 100$. At the left, cartilage shows columns of lacunae and eclls, while, at the right, bone trabeculae and marrow are seen. 
SHADOW.CASTING

PLATE

W. T. DEMPSTER AND R. C. WILLIAMS
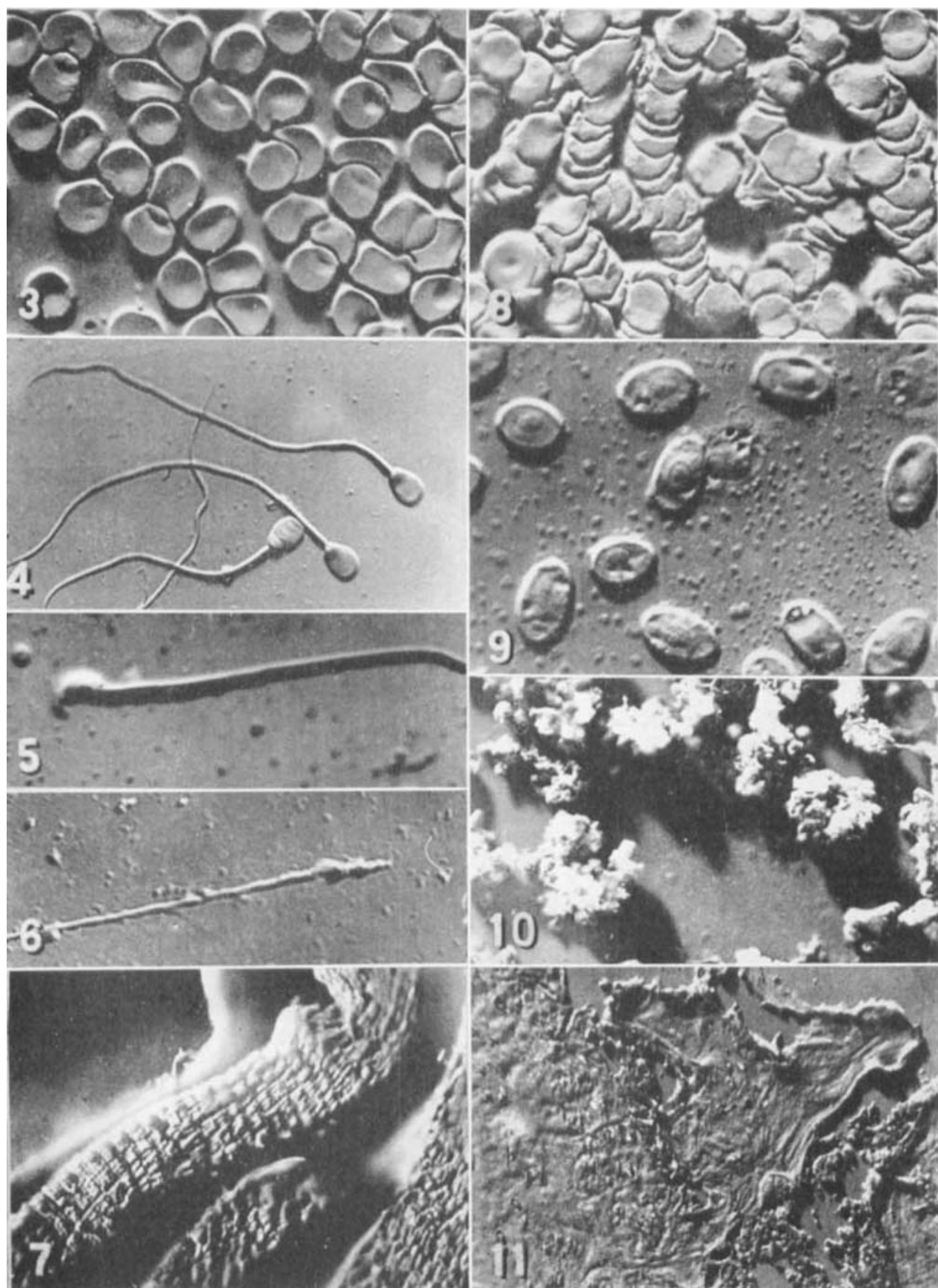

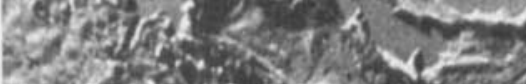

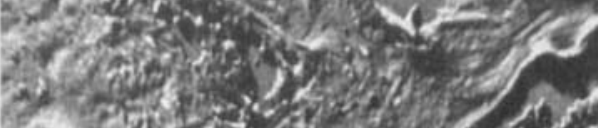

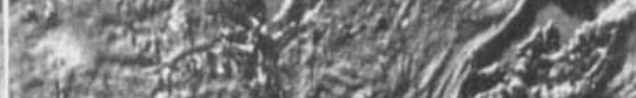

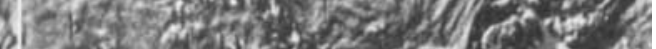

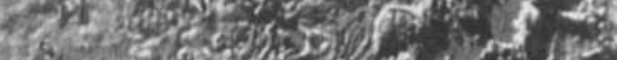

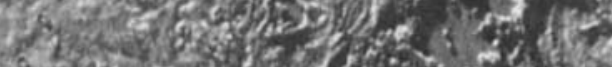

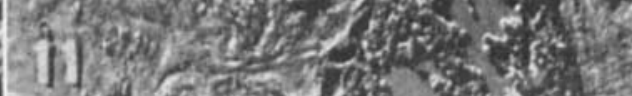

